\title{
Genomic complexity and targeted genes in anaplastic thyroid cancer cell lines
}

\section{Eleanor L Woodward', Andrea Biloglav', Naveen Ravi', Minjun Yang', Lars Ekblad2, Johan Wennerberg ${ }^{3}$ and Kajsa Paulsson 1}

1Division of Clinical Genetics, Department of Laboratory Medicine, Lund University, Lund, Sweden 2Division of Oncology and Pathology, Clinical Sciences, Lund University and Skåne University Hospital, Lund, Sweden ${ }^{3}$ Division of Otorhinolaryngology/Head and Neck Surgery, Clinical Sciences, Lund University and Skåne University Hospital, Lund, Sweden
Correspondence should be addressed to $\mathrm{K}$ Paulsson

Email

kajsa.paulsson@med.lu.se

The authors and journal apologise for an error in the above paper, which appeared in volume $\mathbf{2 4}$ part 5, pages 209-220. The error relates to the reported GEO accession number given on page 211 in the 'RNA sequencing' section.

The original paper stated:

'The data has been submitted to the Gene Expression Omnibus database (GEO; https://www.ncbi.nlm.nih.gov/geo) and is available under accession number GSE94465.'

This should have stated:

'The data has been submitted to the Gene Expression Omnibus database (GEO; https://www.ncbi.nlm.nih.gov/geo) and is available under accession number GSE94464.' 